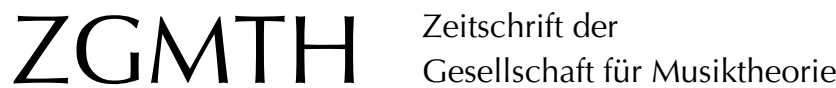

Lewandowski, Stephan / Ong, Arvid (2014): Über erfolgreiche und erfolglose Musik.

Bericht zur IX. Weimarer Fachtagung Musiktheorie und Hörerziehung, Weimar 6.-8.

März 2015. ZGMTH 11/2, 271-274. https://doi.org/10.31751/747

(C) 2014 Stephan Lewandowski, Arvid Ong

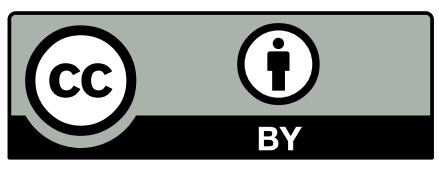

Dieser Text erscheint im Open Access und ist lizenziert unter einer Creative Commons Namensnennung 4.0 International Lizenz.

This is an open access article licensed under a

Creative Commons Attribution 4.0 International License.

veröffentlicht / first published: 24/07/2015

zuletzt geändert / last updated: 19/02/2016 


\section{Über erfolgreiche und erfolglose Musik - Bericht zur IX. Weimarer Fachtagung Musiktheorie und Hörerziehung, Weimar 6.-8. März 2015}

"Warum ist Musik erfolgreich?» Diese provozierende Fragestellung war das Motto der IX. Weimarer Fachtagung Musiktheorie und Hörerziehung vom 6. bis 8. März 2015 - provozierend deshalb, weil die naheliegende Vermutung, es werde um das Aufspüren des Erfolgsrezepts zur Kreativität spar excellence، gehen, das Tagungsthema durchaus ambitioniert erscheinen ließ. Tatsächlich, und dies klang bereits in den Grußworten des Rektors der Weimarer Musikhochschule Christoph Stölzl an, diskutierte ein großer Teil der Tagungsbeiträge die Frage in genau entgegengesetzter Richtung: 'Warum bleibt so manche Musik erfolglos oder gerät in Vergessenheit?

Felix Diergarten (Basel/Luzern) eröffnete den Reigen der Fachbeiträge mit einem Referat über Mozarts Zauberflöte, eine bereits unmittelbar nach ihrer Entstehung nicht nur in Weimar außergewöhnlich erfolgreiche Oper. Den gedanklichen Ausgangspunkt zu diesem Werk und seiner Behandlung im Gehörbildungsunterricht bildeten für Diergarten die saltes und die sneue Skalar, die Albrechtsberger in seinen theoretischen Schriften beschreibt. Es handelt sich dabei um Harmonisierungen der Tonleiter, die identisch sind mit dem heute als , (Dur-Moll-) Parallelismus «ezeichneten Modell (»alte Tonleiter«) bzw. um die Oktavregel (»neue Tonleiter»). Mithilfe eines anschaulich präsentierten Setzkastens voller analytischem Handwerkszeug in Form von Modellhaftigkeiten erläuterte Diergarten, auf welche Weise er damit im Unterricht schnell bis hin zu Echtzeit-Höranalysen gelange. Er wies darauf hin, dass dieses Vorgehen nicht zuletzt als eine pragmatische Reaktion auf die vorhandene Begriffspluralität zu verstehen sei, der sich, bedingt durch viele Nationalitäten und musiktheoretische Traditionen, besonders Lehrende an Musikhochschulen im Unterrichtsalltag häufig gegenübergestellt sehen.
Maren Wilhelm (Leipzig) hielt im Anschluss daran einen Vortrag über den in Deutschland wenig rezipierten französischen Komponisten Gabriel Fauré. Anhand des ersten Satzes aus dem Klavierquintett op. 89 erläuterte sie die Tonsprache Faurés und ging dabei insbesondere auf die wechselseitige Durchdringung von Modalität und Tonalität in seinen Werken ein. Ihre funktionstheoretische Analyse förderte zunächst Besonderheiten und überraschende Momente zutage, die sich als Normabweichungen im Vergleich zu standardisierten harmonischen Wendungen verstehen lassen. Im zweiten Teil ihres Referats stellte sie den Personalstil Faurés als Schnittstelle zwischen (oft linear-kontrapunktisch orientierter) tonaler Satzweise einerseits und präimpressionistischer Klanglichkeit andererseits heraus.

Jens Marggraf (Halle) spürte in seinem Beitrag über Karol Szymanowskis Klavierstück Insel der Sirenen aus dem Zyklus Métopes op. 29 den harmonischen Strategien der Verführung nach. Marggraf trennte dabei Kompositionstechnik und Rezeption. Das ,Verführerische der Komposition liege in der Balance zwischen reichen, komplexen und dabei intuitiven Strukturen auf der einen Seite und der haptischen Virtuosität andererseits. Diese Kombination sei zugleich das, was einer breiteren, über Polen hinausgehenden Rezeption im Wege gestanden habe: Szymanowskis Stil sei kaum eindeutig einzuordnen und schwer zu spielen. Eine weitere These des Vortrags war, dass die undankbare Position Szymanowskis, der szweitberühmteste`Komponist Polens nach Chopin zu sein, ein Problem für den nachhaltigen Publikumserfolg gewesen sei. Szymanowski scheint somit ein Beleg für die Ungleichung zwischen Qualität und Erfolg zu sein.

Eine Abendveranstaltung mit Studierenden der Weimarer Musikhochschule beschloss 
den ersten Tag der Fachtagung. Teilnehmerlnnen eines Spezialkurses des Weimarer Zentrums für Musiktheorie unter der Leitung von Stephan Lewandowski befassten sich mit das Tagungsthema aufgreifenden Fragestellungen: Warum wurde bestimmte Musik niemals erfolgreich bzw. warum geriet erfolgreiche Musik in Vergessenheit? Die Studierenden referierten über vergessene oder zumindest selten aufgeführte Sinfonien von Johann Gottfried Vierling, Anna Amalia, Franz Lachner, Joachim Raff und Heinrich Dorn. Ihre Arbeit reichte dabei von der Quellenforschung bis hin zur Erstellung des Klavierauszugs einer Vierling-Sinfonie, der anstelle einer vorhandenen CD-Einspielung vorgetragen wurde. Das Engagement der Studierenden und die Professionalität der Veranstaltung fanden allgemeine Anerkennung.

Florian Kleissle (Weimar/Dresden) eröffnete den zweiten Tag mit einem Vortrag zu Sequenzmodellen in Johann Sebastian Bachs Orgelfugen. Er stellte hierzu eine umfassende systematische Katalogisierung dieser Modellhaftigkeiten vor. In der anschließenden Diskussion wurde besonders herausgestellt, dass nicht die satztechnischen Modelle selbst als wiederkehrende Topoi, sondern der individuelle Umgang mit ihnen entscheidend für den Personalstil eines Komponisten sei - und damit auch maßgeblich für dessen nachhaltigen Erfolg. Interessant war ebenfalls die teilweise von Florian Kleissle gebotene kombinatorische Herleitung einzelner Satzmodelle, so etwa das Vorhandensein eines Fauxbourdonsatzes als Bestandteil von Quintfallsequenzen.

Raphael Thöne (Hannover) zeigte in einem workshop-artig angelegten unterhaltsamen Beitrag die Möglichkeiten professioneller Orchester-Sample-Mockup-Erstellung im Film- und Eventbereich. Dabei demonstrierte Thöne, wie mit dem Programm Cubase 7.5 in Kombination mit Soundbanken wie Vienna Symphonic Library und LA Scoring Strings verblüffend realistische Klangergebnisse erzielt werden können. Es wurde jedoch auch eine Entwicklung deutlich, die das Berufsbild des Komponisten, im Speziellen des Filmkomponisten, nachhaltig verändert: Die Ver- marktungsmöglichkeiten von Musik hängen Thöne zufolge immer stärker vom Exposé ab. Die heutigen Möglichkeiten, mit begrenztem Equipment und kleinem Budget fertige Klangbeispiele herzustellen, steigern demnach die Erwartung, auf begrenzte klangliche Vorstellungskraft von Auftraggebern (insbesondere Regisseuren) Rücksicht zu nehmen. Im Filmmusikgenre sei somit 'Sounddesign ‘ die zentrale Kompositionsdisziplin, auch wenn satztechnisch-handwerkliche Grundfertigkeiten aus der Sicht Thönes nach wie vor eine wichtige Basis bildeten. Der Filmmusikkomponist müsse zwei Aspekte vereinen: technischen Sachverstand und eine sungehemmte Kreativität. Im Anschluss an den Beitrag entspann sich eine Diskussion über das Thema Mensch - Computer: Möglichkeiten und Grenzen.

Nach der Mittagspause schloss sich ein Workshop mit dem Titel Analyse und Interpretation an, der auf vielfach geäußerten Wunsch während der letztjährigen Weimarer Fachtagung nun realisiert wurde. Als Referent konnte kein geringerer als Peter Gülke, erst kürzlich mit dem Siemenspreis ausgezeichneter Ehrendoktor und Ehrensenator der Weimarer Musikhochschule, gewonnen werden. Geprobt wurde Ludwig van Beethovens Streichquartett op. 18, Nr. 4. Die hervorragend eingespielte Streichquartett-Formation bestand aus Elisabeth Marasch und Eva-Lotta Baumann (Violinen), Fabian Lindner (Viola) und Sophia Marie Garbe (Violoncello). Die Probe wurde an einigen Stellen ergänzt durch Kommentare Gülkes zur Entstehungsgeschichte des Werks sowie zu einigen analytischen und aufführungspraktischen Aspekten.

Von einer völlig anderen Seite näherte sich Gesine Schröder (Wien/Leipzig) dem Tagungsthema. Sie befasste sich mit einem Stück Geschichte der Musik und Musiktheorie in der DDR. Anhand des Komponisten Kurt Schwaen beleuchtete sie die Auswirkungen des politischen Umfeldes auf das künstlerische und kunsttheoretische Schaffen. Dabei stellte sie einige Paradoxien der musikalisch-ästhetischen Debatte der DDR heraus. So beispielsweise den Anspruch an die Gegenwartsmusik, Musik für das Volk zu 
sein (im Gegensatz zu der als formalistisch gebrandmarkten Musik des Westens), sich aber auch von der Volksmusik des Dritten Reichs abzugrenzen. Schwaen habe als Idealist der ersten Stunde mit seiner Schrift Tonweisen sind Denkweisen - Beiträge über die Musik als gesellschaftliche Funktion von 1949 insbesondere in theoretischer Hinsicht ein Standardwerk für das politische System geliefert. Er habe sich jedoch in einzelnen Aspekten seine künstlerische Identität bewahrt. So seien sogenannte Massenlieder für ihn ästhetisch uninteressant gewesen, auch wenn diese dem sozialistischen Ideal näher gestanden hätten als die sneue، Modalität Schwaenscher Lieder.

Jörn Arnecke (Weimar) stellte seine Kinderoper über Astrid Lindgrens Ronja Räubertochter vor, die erst kürzlich an der Deutschen Oper am Rhein Düsseldorf-Duisburg Uraufführung feierte. Selten bieten Komponisten derart tiefe und offene Einblicke in ihr Schaffen und die Überlegungen im Umfeld der Entstehung eines Werks. Arnecke beschrieb, jenseits aller rein kompositorischen Überlegungen, Erwartungshaltungen von Beteiligten gegenüber Opernprojekten als eine Art ımagisches Dreieckı zwischen Auftraggebern, Ausführenden und Verlag, in dessen Zentrum der Komponist stehe, der seine künstlerischen Ideen umsetzen möchte. Das Publikum als umspannende Instanz bilde nicht zuletzt den quantitativen Gradmesser für Erfolg. Arnecke machte somit deutlich, dass es das eine Erfolgsrezept nicht geben könne, da auch der nicht planbare Faktor `Glückı eine entscheidende Rolle spiele.

Gisela Schreiber (Halle/Saale) stellte ihre Konzeption für Musiktheorieunterricht an der Hallenser Musikschule vor. Sie verfolgte in ihrem Vortrag konsequent ein progressives Programm satztechnischer Übungen und Gehörbildungsaufgaben, das den Anwesenden einen offenen Einblick in ihr Unterrichten bot. Mit diesem Angebot sei es ihr gelungen bereits zahlreiche SchülerInnen erfolgreich auf Aufnahmeprüfungen vorzubereiten oder Jugendlichen musikalisches Verständnis zusätzlich zum Instrumental- oder Gesangsunterricht zu vermitteln. Am Rande entspann sich im Anschluss ihres Beitrags eine Diskus- sion über den zugrundeliegenden Kanon im vorhochschulischen musiktheoretischen Ausbildungsbereich.

Besonders das letztgenannte Thema wurde anschließend vertieft in einem Streitgespräch zur Frage: Musiktheorie für Jugendliche - vom Aussterben bedroht? Das Gespräch leitete Kai Martin (Weimar), weitere Teilnehmende waren Gisela Schreiber (Halle/Saale), Eugenie Erhard (Weimar), Arvid Ong (Detmold/Hannover) und Stephan Lewandowski (Weimar/ Dresden). Obwohl die Diskussion inhaltlich viele Teilbereiche dieser umfangreichen Thematik nur streifen konnte, gelang dennoch eine Sensibilisierung der Anwesenden dafür, dass viele der Veränderungen der letzten Jahre innerhalb der Disziplin Musiktheorie - etwa der Wandel zu einem verstärkt historisch-informiertem Fach, aber auch, nicht selten dadurch bedingt, neu entstandene methodischdidaktische Ansätze - für den schulischen und musikschulischen Alltag kaum relevant geworden sind.

Karl Traugott Goldbach (Kassel) referierte über den Komponisten Louis Spohr, der zu Lebzeiten vor allem in England frenetisch gefeiert wurde. Dass dieser in Vergessenheit geraten sei, hätte, so die These des Vortragenden, vor allem drei Ursachen. Erstens: Spohrs Musik sei immer außerhalb jeglicher Modeerscheinungen der Zeit gestanden. Die Veränderungen um 1850, so erscheine es, habe er kompositorisch nicht nachvollziehen wollen, obwohl seine stilistischen Eigenheiten ihn auch nicht als biedermeierlichen Traditionalisten auszeichneten. Zweitens: Einen nicht unwesentlichen Anteil an der ungünstigen Rezeption Spohrs hätte die zeitgenössische Rezension gehabt. Die ausgeprägte Chromatik, welche beispielsweise bei Wagner als typischer Personalstil verstanden worden wäre, hätte man Spohr als Manierismus ausgelegt. Hinzu komme aber noch ein dritter Aspekt: Die mit Spohrs Werken verbundenen aufführungspraktischen Probleme. Instrumentatorische Eigenheiten und technisch-virtuose Schwierigkeiten trügen, so Goldbach, dazu bei, dass zahlreiche Werke Spohrs heute selten zu hören seien. 
Kitschkomponist oder Epigone lautete der Titel des Beitrags von Juliane Brandes (Freiburg im Breisgau), welcher sich mit dem Komponisten und Musiktheoretiker Ludwig Thuille befasste. Thuilles Tonsprache im Umfeld der Münchner Schule sei zunächst durch Brahms beeinflusst gewesen, spätestens ab 1886 habe sich Thuille aber neudeutschen Strömungen zugewandt. Die Musik enthalte eine Fülle von Anspielungen auf musikalische Idiome des späten 19. Jahrhunderts. Dies mache ihn gewissermaßen zu einem Prototyp der Kompositionstechnik dieser Epoche. Der Theoretiker Thuille sei ein Beleg dafür, dass die Partimentotechnik in dieser Zeit immer noch gegenwärtig gewesen sei. Brandes skizzierte die Bedeutung von Satzmodellen wie der `Oktavregelı, deren modifizierte Erscheinungsformen sie bei Thuille nachwies. Das Klischeehafte insbesondere am Beispiel der Oper Lobetanz entfachte darüber hinaus eine
Diskussion über die Frage nach (Selbst-)Ironie bei Thuille.

Johannes Kretschmer (Weimar) beschäftigte sich mit dem Werk der Komponistin Sofia Gubaidulina, wobei er das Ineinandergreifen von exakt konstruierten, nicht selten mathematischen Reihen folgenden Abschnitten sowie intuitiv komponierten Passagen herausstellte.

Nach einem mittäglichen Schlussplenum, in dem sich die Organisatoren der Fachtagung, Jörn Arnecke, Maren Wilhelm und Jens Marggraf, bei allen ReferentInnen und Teilnehmenden bedankten sowie einen Ausblick auf die zehnte Tagung im kommenden Jahr gaben, konnte als abschließendes Resümee in aller Knappheit festgestellt werden: Auch wenn nicht alle Fragen zu Erfolg oder Misserfolg von Musik geklärt wurden, die Tagung selbst war erfolgreich.

Stephan Lewandowski, Arvid Ong 